\title{
A rare case of superior mesenteric vein trombosis in pregnancy
}

\author{
Pooja Chandak ${ }^{1, *}$, Shobha Toshniwal ${ }^{2}$, Sameer Deshmukh ${ }^{3}$ \\ ${ }^{1}$ Resident, ${ }^{2}$ Private Practitioner, ${ }^{\mathbf{3}}$ Intern Doctor, Dept. of Obstetrics and Gynaecology, ${ }^{\mathbf{1}, \mathbf{3}}$ Government Medical College, Nanded, \\ Maharashtra, ${ }^{2}$ Toshniwal Chest Hospital \& Maternity Home, Nanded, Maharashtra, India \\ *Corresponding Author: Pooja Chandak \\ Email: drpoojachandak@gmail.com
}

Received: $13^{\text {th }}$ September, 2018

Accepted: $17^{\text {th }}$ September, 2018

\begin{abstract}
Superior mesenteric venous thrombosis is a rare entity in itself with pregnancy being the cause is a rare possibility. Here presented a rare case of 25 years primigravida with term gestation with acute abdomen underwent LSCS for suspicion of ruptured uterus suggested ischemic bowel segment due to SMV thrombosis who underwent resection and anastomosis with uneventful post op period.
\end{abstract}

Keywords: Superior mesenteric vein thrombosis, Pregnancy.

\section{Introduction}

Small bowel ischemia is a relatively uncommon disorder; the primary causes are diverse and can be grouped into five major categories: (1) strangulation, (2) low-flow states (arrhythmia, sepsis, shock), (3) embolus or thrombosis of superior mesenteric artery, (4) superior mesenteric vein thrombosis (MVT), and (5) miscellaneous. Due to vague clinical presentation and the lack of specific diagnostic tests, early diagnosis for intestinal ischemia is difficult, resulting in significant morbidity and mortality. We herein report a case of a 25 -year-old woman who was presented with the signs and symptoms of latent labor at 38 weeks gestation. Emergency exploratory laparotomy done for the patient revealed gangrene of small bowel without mechanical obstruction. Hypercoagulable state is normally found in pregnant women, which was believed to result in superior MVT and then intestinal ischemia.

\section{Case Report}

A $25 \mathrm{yrs}$ old primigravida with 38 weeks gestation c/o pain in abdomen and 2 to 3 episodes of vomiting patient was admitted and on arrival her pulse was $92 \mathrm{bpm}$ and bp 110/70mmhg with per abdominal examination suggestive of single live full term intrauterine gestation with per vaginal examination s/o latent labor. urine albumin was done to r/o preeclampsia as patient was c/o vomiting s/o nil albuminuria patient started on low dose Pitocin drip i.e. 2.5 units in 1 pint RL @ 8 drops/min. As the labor progressed within 4 hours patient developed features of acute abdomen with severe pain in abdomen with shoulder tip pain, breathlessness tachycardia, hypotension and distension of abdomen with absent fetal heart sound an USG done on emergency basis to r/o uterine rupture s/o an intrauterine fetal death with uterine margins intact with dilated bowel loops and minimal free fluid in peritoneal cavity.
Laboratory investigations suggestive of only leukocytosis with WBC count of $18000 / \mathrm{mm} 3$ with all the other blood investigations being normal.

A decision for emergency exploratory laparotomy taken as patient's general condition was worsening with suspicion of ruptured uterus, patient started on ionotropic support and the procedure done under general anesthesia. Abdomen opened by pfannenstiel's incision. Intraoperative findings suggestive of intact uterus with gangrenous small bowel segment of around $60 \mathrm{cms}$ with $500 \mathrm{cc}$ of serosanguinous ascitic fluid. The pregnancy was terminated by LSCS a fresh still born male baby of $2.5 \mathrm{~kg}$ delivered and there was no evidence of any retro-placental clot. Intra-operative call was given to General surgeon who did resection of gangrenous bowel segment and primary anastomoses. 1 pint blood transfused intraoperatively. Abdomen closed after keeping an intra-peritoneal drain and patient was shifted to surgical ICU postoperatively with continued ionotropic support which was withdrawn on postoperative day 3 . The resected bowel segment was sent for HPR. Patient was kept NBM for 5 days and started on oral sips on day 6 once the bowel sounds occurred. Patient given higher antibiotics and anticoagulant therapy with Injection Heparin unfractionated 5000IU 6 hourly for 5 days with warfarin overlap from day 3 with $2 \mathrm{mg}$ tablet to maintain an International Normalized Ratio (INR) between 2 to 3 . Sutures removed on day 10. Patient discharged from the hospital on day 12 and called for follow up after 15 days with HPR report. Other investigations to rule out other causes for thrombosis were not done as the patient was not affording.

HPR report showed necrotic changes with denudation of bowel mucosa with inflammatory changes with neutrophilic infiltration with edema all over. 


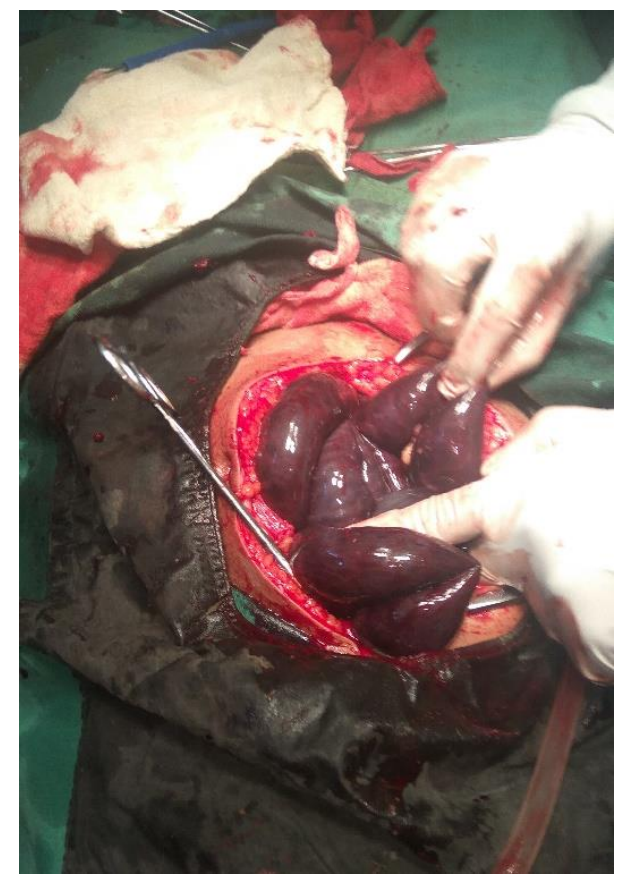

Fig. 1

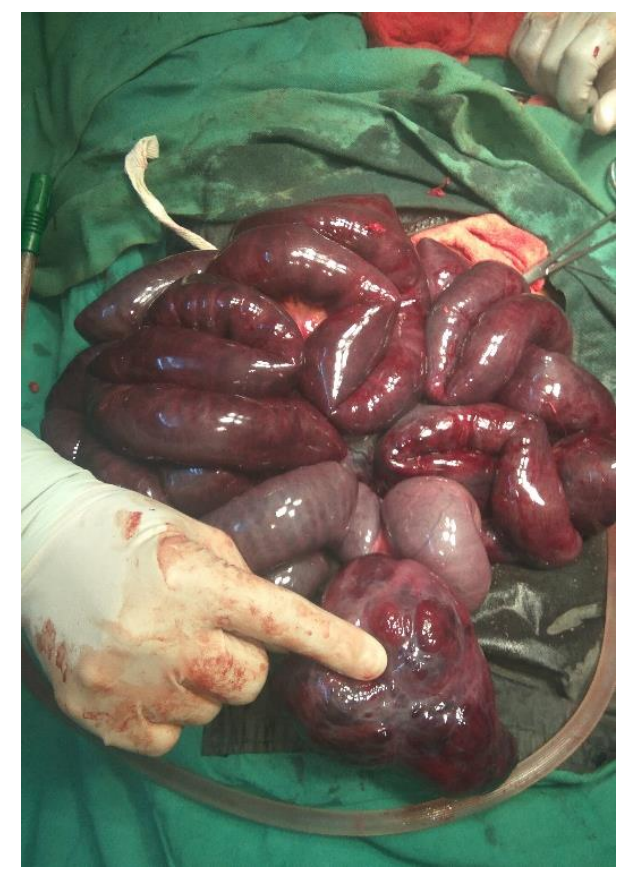

Fig. 2

\section{Discussion}

Superior mesenteric vein drains blood from small bowel specifically from jejunum and ileum, it joins with the splenic vein behind the neck of pancreas to form hepatic portal vein. Its thrombosis is a rare entity and responsible for 5 to $15 \%$ cases of mesenteric ischemia. After searching the different articles only 17 cases of superior mesenteric venous thrombosis with pregnancy being the cause for venous thrombosis have been reported. The causes SMV thrombosis include hypercoagulable states like protein $\mathrm{C}$ and protein $\mathrm{S}$ deficiency, Factor 5 Leiden Mutation, antithrombin 3 deficiency, polycythemia vera, neoplasms, abdominal trauma, intra-abdominal infections \& inflammatory causes like pancreatitis, ulcerative colitis, Chron's disease with pregnancy being the rarest cause. Idiopathic or primary cause accounts for 21 to $49 \%$ cases. Use of oral contraceptive pills can enhance the chances of thrombosis in the patient but this patient was not using any hormonal contraception. In acute cases it produces symptoms of acute abdomen and in chronic cases it produces symptoms of portal hypertension. Acute cases produce non-specific symptoms like severe pain in abdomen, distension, vomiting, bloating, malena, hematemesis and hematochezia, fever, tachycardia and hypotension hence in pregnancy, the diagnosis of MVT is difficult because some symptoms can wrongfully present as normal changes of pregnancy. Hence high index of suspicion required for correct diagnosis. Contrast enhanced CT or MRI have high sensitivity for diagnosing the condition. But the colour dopller being the cheapest and readily available investigating modality remains the $1^{\text {st }}$ investigation of choice and has the sensitivity of $70 \%$ to diagnose the condition.

Exploratory laparotomy and resection and anastomosis is the treatment for this acute entity if peritoneal symptoms develop. In the absence of acute symptoms conservative treatment in the form of antibiotics and IV hydration along with anticoagulation treatment can be given.

Anticoagulant therapy is the cornerstone for managing this condition along with surgical or conservative management. Low molecular weight heparin in the doses of $0.6 \mathrm{mg} / \mathrm{kg}$ or unfractionated heparin in the dose of 5000IU6 hourly followed by overlapping with warfarin to maintain INR between 2 to 3 is the treatment of choice. There are no defined guidelines for the duration of anticoagulation and the decision is to be made as per the case.

The decision on continuation or termination of pregnancy is taken as per the case and available facilities. While workers like Engelhardt TC, et al. and Fouada $\mathrm{M}$ A, et al. successfully continued the pregnancy, Lin $\mathrm{H}$, et al. and FOO E, et al. terminated the pregnancy.

\section{Conclusion}

To conclude, we stress that in a pregnant woman with a clinical presentation of acute abdomen, even with the absence of the other known risk factors, superior mesenteric vein thrombosis should be kept in mind and included in the differential diagnosis to avoid potential complications. 


\section{References}

1. Shui LH, Rafi J, Corder A, Mowbray D. Mid-gut volvulus and mesenteric vessel thrombosis in pregnancy: case report and literature review. Archives of Gynecology and Obstetrics. 2011;283(supplement 1):S39-S43.

2. Engelhardt TC, Kerstein MD. Pregnancy and mesenteric venous thrombosis. Southern Medical

Journal. 1989;82(11):1441-1443.

3. Atakan Al R, Borekci B, Ozturk G, Akcay MN, Kadanali $\mathrm{S}$. Acute mesenteric venous thrombosis due to protein $\mathrm{S}$ deficiency in a pregnant woman. Journal of Obstetrics and Gynaecology Research. 2009;35(4):804-807.

4. C. M. Chan, W. L. Chen, J. H. Chen, Y. L. Wu, and C. C. Huang. "Pregnancy-induced acute intestinal infarction in a woman with chronic idiopathic mesenteric vein thrombosis under regular anticoagulation treatment." Medical Principles and Practice. 2009;18(5):422-424.

5. Ha HK, Rha SE, Kim AY, Auh YH: CT and MR diagnoses of intestinal ischemia. Semin Ultrasound CT MR. 2000;21:40-55.

6. Augustin G, Majerovic M. Non-obstetrical acute abdomen during pregnancy. Eur J Obstet Gynecol Reprod Biol. 2007;131(1):4-12.
7. Fouada M A, Pathania A G, Marouf R. Primary mesenteric venous thrombosis in a 28 -week pregnant woman. Med Principles Pract. 2001;10:204-6.

8. Taourel PG, Deneuville M, Pradel JA, Regent D, and Bruel JM (1996) Acute Mesenteric Ischemia: diagnosis with contrast enhanced CT. Radiology. 199:632.

9. Kozuch PL, Brandt LJ. Review article: diagnosis and management of mesenteric ischaemia with an emphasis on pharmacotherapy. Aliment Pharmacol Ther. 2005;21(3):201-15.

10. Acosta S, Alhadad A, Svensson P, Ekberg O. Epidemiology, risk and prognostic factors in mesenteric venous thrombosis. Br J Surg. 2008;95(10):1245-51.

How to cite this article: Chandak $\mathrm{P}$, Toshniwal S, Deshmukh S. A rare case of superior mesenteric vein trombosis in pregnancy. Indian J Obstet Gynecol Res. 2018;5(4):576-578 